\title{
Building the Future with Envisioning
}

\author{
Stuart Reeves I University of Nottingham I stuart@tropic.org.uk \\ interactions, Jan/Feb 2013, ACM Press
}

HCI and related interaction design fields often explore the challenges of future technologies. This future orientation is intrinsic to ubiquitous computing. Conceived during the late 1980s and early 1990s, and developed initially through the work of Xerox PARC's Computer Sciences Lab (CSL), ubiquitous computing (ubicomp) set out a compelling vision for the future and a strategic path by which to get there.

In 1991, CSL's manager, Mark Weiser, presented the ubicomp vision in a Scientific American article called "The Computer for the 21st Century" [1]. Although it appeared in a popular science magazine, the article has become one of the foundational references in the ubicomp literature. A key driver of its success was its radical contrast with then popular future visions of immersive virtual realities. Through an evocative fictional scenario, Weiser depicted "what it would be like to live in a world full of invisible widgets." In introducing this scenario, Weiser identified that it was not enough to describe ubicomp only in terms of its principles and technologies.

The article's "Sal scenario" employed fiction to link present and future in a way that was seamless and striking, yet familiar. While the scenario may seem utopian in its sensibilities, it nevertheless highlighted the intrinsic importance of social interaction in technology design:

"Sal awakens: she smells coffee. A few minutes ago her alarm clock, alerted by her restless rolling before waking, had quietly asked 'coffee?' and she had mumbled 'yes.' 'Yes' and 'no' are the only words it knows.

Sal looks out her windows at her neighborhood. Sunlight and a fence are visible through one, but through others she sees electronic trails that have been kept for her of neighbors coming and going during the early morning. Privacy conventions and practical data rates prevent displaying video footage, but time markers and electronic tracks on the neighborhood map let Sal feel cozy in her street.

Glancing at the windows to her kids' rooms she can see that they got up 15 and 20 minutes ago and are already in the kitchen. Noticing that she is up, they start making more noise."

So striking was this piece of writing that it became perhaps the most cited article within technical ubicomp research, in spite of its provenance as a piece for nontechnical laypersons.

The compelling mixture of fictional elements, coupled with extrapolations from existing technological capabilities and apparent forecasts of future societal situations, is called envisioning [2]. Envisioning is a self-referential process that looks toward the future for both inspiration and challenge in deciding what digital technologies to design and build next. In mixing future-oriented features such as fiction and extrapolation, envisioning imagines possible societies and possible technologies. On the one hand, today's technological capabilities are extrapolated 
into the future, for instance by considering the implications of ever-increasing processing power and storage capabilities coupled with reductions in size and cost. These extrapolations become enablers of envisioning what a future society could look like (Figure 1a). Prospective societal situations then provide the sorts of novel scenarios in which these extrapolated technological capabilities can find their use (Figure 1b). In this interaction, the looping envisioning process is informed by a background of historical technological and societal "trends" (Figure 1c).

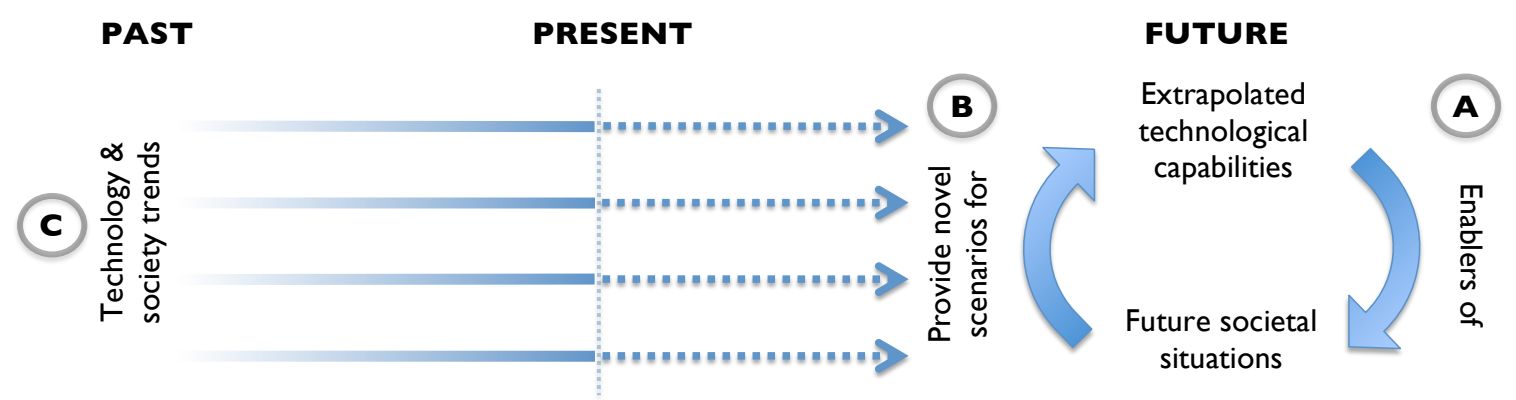

Figure 1. Some key features of envisioning.

While mixing in fictional elements supports envisioning's communication and accessibility, fiction also helps construct a greater range of possible interpretations than would be afforded by traditional "factual" forecasts. However, because of this open character, envisionings are often interpreted as forecasts, regardless of whether the author intended the envisioning to contain any element of forecast (indeed, this is typically not their purpose, as we shall see later).

\section{Envisionings Make Disappointing Forecasts}

The HCI and ubicomp communities have spent much time following through on the implications of the ubicomp envisioning, making progress on a set of canonical subfields drawn more or less directly from the technological situations and societal needs found in the original envisioning. These include power and energy management, context awareness, perceptual invisibility, software adaptation, mobility, networking issues, novel interaction techniques, location-tracking systems, and privacy issues.

The ubicomp envisioning itself is often assumed in some ways to be inevitable [3]. As noted earlier, we tend to treat envisionings as forecasts- that is, in terms of criteria related to their reliability, accuracy, precision, and achievability (e.g., how likely we are to end up in the world that the envisioning describes) — even though these sorts of criteria may not be applicable to envisioning in the first place. More broadly, there are a number of ways we tend to misunderstand the nature and purpose of envisioning.

First, futures cannot be determined by forecasts of technological advancements. Many envisionings rely upon Moore's Law-that is, exponential growth in transistor counts and chip performance. However, this is often treated as a natural law with predictive power instead of as a self-fulfilling manufacturing target that is achieved, rather than inevitable. The net result can be a kind of technological tunnel vision 
whereby Moore's Law-influenced forecasts imply dominance of a single road to the future.

Second, futures are necessarily limited by what we have previously experienced. In Schütz's phenomenological sociology, he explores how members of society develop stocks of common-sense knowledge of the social world through past and present everyday experiences [4]. The problem for members of society is how we act on a future that is not here yet. To do this he argues that we construct (and act on) a picture of the future in the form of an "anticipated hindsight," i.e., one that is projected forward, then transposed back to the present, in order to inform how we will come to experience that future moment. Naturally, this limits our thinking about what societal situations may be possible, as well as what future technologies we can, might, or will build, and what the impacts of those technologies can, might, or will be. Envisionings are, and can only ever be, deeply embedded in the past, restricting the very dimensions of feasibility, possibility, and so on. This means ubicomp's envisioning can present us only with a set of choices configured by the limitations of the past. Envisioning often becomes a struggle against the blandness of these limitations.

Third, the history of computing is littered with a range of envisionings that in hindsight come to be seen as "incorrect" when measured up to subsequent developments. Envisionings as forecasts, viewed historically, have offered a disappointing sense of broken promises, such as in the anticipations of artificially intelligent systems or the excitement around the opportunities of immersive virtual reality. In the light of this historical perspective, we notice that many significant developments in the history of computing were not envisioned-for instance, the Web developed as a workplace tool for CERN scientists [5].

\section{Envisionings as Useful Fictions}

These issues should concern us if we try to use envisionings as strategic forecasts and predictions. If we do this, then aligning the envisioning-as-likely-future with the world as we know it becomes problematic. Indeed, the ubicomp envisioning has periodically been subject to some rethinking of what was originally meant by Weiser and CSL [6], and concerns have been expressed over whether the ubicomp envisioning matches reality [7]. In this vein, Genevieve Bell and Paul Dourish argue that ubicomp as a field has continually aimed for yesterday's vision of the future and tended to miss what is already here [8]. In contrast with this widespread attitude, I think we need to look at what work the idea of ubicomp was and is doing for us, and in what ways envisionings provide useful fictions rather than disappointing forecasts.

Envisioning and its uses are organizational. The early ubicomp envisioningparticularly Weiser's Scientific American article-serves as a representation of the relationship between CSL, PARC, and their parent company, Xerox. During this period, Xerox was changing the way it faced the world, moving from "the photocopier company" to "the Document Company" [9]. The ubicomp envisioning was developed in that particular context, sensitive to those particular organizational changes (e.g., the famous pads, tabs, and boards can be seen as a response to the need for diverse document production and consumption devices). It was also reacting to another envisioning: virtual reality. Presenting the envisioning to a popular science audience was also presenting it to Xerox, and through this encapsulating a range of organizational purposes. 
These purposes run deeper than just public maintenance of an organizational relationship. John Hughes's ethnographic work at CSL in the mid 1990s lets us further inside the lab during this critical period and elaborates this envisioning as organizational perspective (Hughes, personal communication). Hughes reports how the idea of ubicomp provided an organizing framework across CSL's research, pulling together what were seen as current computing trends and their various implications. The ubicomp envisioning coalesced fragments of the lab's work into a coherent story to perform various roles: as a check against the fragmentary side effects of encouraging a culture of freedom and initiative; maintaining the reputation and pioneering character of PARC and CSL; ensuring distinctiveness and branding; drawing in new recruits; providing a vocabulary and method of coordinating lab work; and engendering a shared sense of direction. With this came a sense of ownership-ubicomp was CSL's vision.

Envisionings are less straightforward than we treat them. They have the character of appearing to have been constructed before certain technological developments. However, they may be constructed in order to both collect a disparate set of technological developments after the fact, and provide goals, plans, and orders for new work. Weiser took CSL's various activities and molded them into a coherent strategic picture of the principles, direction, and meaning of the research.

In this light, Weiser and CSL's efforts seem all the more impressive, given the ubicomp envisioning's invigorating effect across HCI and related fields. A considerable danger for envisionings and their organizational nature is that they can be exercises in risk management: conservative, bland, and boring, but at the same time providing the veneer of "forward thinking" (see the preponderance of flatsurface/glass-based interactions in recent corporate promotional videos). Instead, the ubicomp envisioning gave birth to a whole new field.

There are further dangers (perhaps more readily characterized as trade-offs). As part of being organizationally useful fictions, envisionings help animate and orient the construction of design worlds in which users, scenarios, and settings are invoked in order to work through design decision-making processes [10]. Envisionings help bound and make manageable the apparent limitlessness of design, while providing ways to coordinate work between design collaborators. They ossify, make concrete, and establish paradigms for working within. However, like concrete, they have an oppositional viscosity that hardens over time.

\section{Topicalizing Envisionings}

Unlike explicit formal processes such as using scenarios and personas [11], envisionings are often backgrounded in design work. Instead, we need to foreground and explicate in detail the core envisionings that we, on a daily basis, use, align with, and rely on when designing technologies. When we start talking about the coming Internet of Things, the significance of augmented-reality solutions, or the idea of a future with IT as a utility, we can turn such envisionings into topics of investigation by beginning to question aspects of them, such as:

- What was the context in which the envisioning was created? What issues of the present is the envisioning concerned with and speaking to?

- Who might be the intended audience(s) of the envisioning?

- For what organizational purposes could the envisioning be useful? 
- What happens if the envisioning is read as a useful fiction instead of a forecast or prediction?

- What is the relationship of the envisioning to other, older envisionings? What can we learn from their comparison?

In doing this we will need to reevaluate the assumptions embedded in envisionings. This is in contrast with our normative approach, which seeks to find ever-new ways to reinterpret and align envisionings with changes in technology and society according to criteria for which they were not necessarily intended. We will need to recognize an envisioning's fragilities as well as its strengths. We may choose to stick with them as useful fictions that support organizational purposes, such as tools for collaborative work and ideation. In the end, envisionings should help us stimulate the generation of novel ideas, rather than invite evaluations of how well they fit to reality.

We need to engage with envisionings as a design method that plays a fundamental role in the construction of design worlds. We need to pick them rather than fall into using them by default. In selecting a particular envisioning, we become more mindful of what pathways we might be shutting down as possibilities.

Finally, we need more envisionings, rather than fewer. Proliferation challenges any one single envisioning being the de facto standard and presents a guard against a teleological view of technology's role in our world. The ubicomp envisioning itself emerged in part through a reaction to virtual reality's envisioning; envisionings need this kind of dialogue to temper the effects of turning to concrete over time.

\section{Acknowledgments}

Thanks go to Bob Anderson, Robert Dingwall, and Murray Goulden for extensive discussions, comments, ideas, and suggestions that were instrumental in the development and preparation of this article. Thanks also to Erik Stolterman for his input. This work is supported by Horizon Digital Economy Research, RCUK grant $\mathrm{EP} / \mathrm{G} 065802 / 1$.

\section{References}

1. Weiser, M. The computer for the twenty-first century. Scientific American (Sept. 1991), 94-104.

2. Reeves, S. Envisioning ubiquitous computing. Proc. of CHI 2012. ACM Press, New York. 2012.

3. Greenfield, A. Everyware: the Dawning Age of Ubiquitous Computing. Peachpit Press, 2006.

4. Schütz, A. Tiresias, or our knowledge of future events. Social Research: An International Quarterly 26, 1 (1959), 71-89.

5. Berners-Lee, T. Weaving the Web. HarperOne, 1999.

6. Tolmie, P., Pycock, J., Diggins, T., MacLean, A., and Karsenty, A. (2002)

Unremarkable computing. Proc. of CHI 2002. ACM Press, 2002. 
7. Rogers, Y. Moving on from Weiser's vision of calm computing: engaging ubicomp experiences. Proc. of Ubicomp 2006. Springer, 2006, 404-421.

8. Bell, G. and Dourish, P. (2007) Yesterday's tomorrows: Notes on ubiquitous computing's dominant vision. Personal Ubiquitous Computing 11, 2 (2007), 133-143.

9. Collins, G. Xerox attempts a new beginning by making its name the last word in corporate rechristening. New York Times. August 4, 1994, 17.

10. Sharrock, W. and Anderson, R. The user as a scenic feature of the design space. Design Studies 15, 1 (1994), 5-18.

11. Friess, E. Personas and decision making in the design process: An ethnographic case study. Proc. of CHI 2012. ACM Press, New York, 2012, 1209-1218. 